\title{
Identification récursive en transmittance des systèmes mécaniques sous-actionnés (Application au pendule
} inversé)

\author{
Recursive identification in transmittance of under-operated mechanical \\ system (applied to inversed pendulum)
}

\author{
Jacques Kadima Kazaku ${ }^{1}$ \\ ${ }^{1}$ Université de Lubumbashi/Polytechnique/Electromécanique, République Démocratique du Congo, \\ jacqueskazaku@polytechunilu.ac.cd
}

RÉSUMÉ. Une nouvelle approche d'identification des systèmes mécaniques sous actionnés est présentée dans cet article. L'idée de base est d'étendre les algorithmes des moindres carrés étendus et de Steiglitz et McBride au cas des systèmes sous-actionnés. Les modèles considérés sont de type OE et ARMAX. Une évaluation comparative en simulation par des exemples numériques a montré clairement la qualité de l'identification en utilisant cette approche par rapport aux résultats obtenus en utilisant la méthode des moindres carrés linéaires non récursifs. L'utilisation des modèles OE et ARMAX a permis d'améliorer la convergence asymptotique de ces algorithmes.

ABSTRACT. A new approach of identifying mechanical system under-operated is presented in this article. The basic idea is to spread the extensive least-square algorithm and the Stieglitz and McBride algorithm to the case of under-operated systems. The considered models are OE and ARMAX type. The comparative evaluation through simulation using numerical example shows clearly the quality of the identification using this approach compared to the result obtained by the non-recursive linear least-squared methods. The use of the OE and ARMAX models has made the improvement of the asymptotic convergence of these algorithms possible.

MOTS-CLÉS. Identification récursive, transmittance, systèmes mécaniques sous-actionnés.

KEYWORDS. Recursive Identification, Transmittance, Under-operated mechanical systems.

\section{Introduction}

L'automatique consiste à étudier des systèmes réels de différentes disciplines (électronique, mécanique, thermique, chimie, écologie,...) en vue de les analyser, les surveiller, les diagnostiquer, et les commander. Cela nécessite la disponibilité d'un modèle mathématique de ce système réel. Un système est un objet dans lequel des variables de différents types interagissent et produisent des signaux observables. Lors que le modèle du système n'est pas connu, il est nécessaire de procéder à son identification. Ce modèle ne cherche qu'à reproduire "au mieux" un fonctionnement dynamique dans un contexte donné sans se préoccuper de la signification physique éventuelle des paramètres dont il dépend.

L'identification consiste à chercher les paramètres de modèles mathématiques d'un système, à partir de données expérimentales et de connaissances disponibles a priori. Ces paramètres peuvent avoir une signification physique, comme dans les modèles de connaissance (issus de lois de la mécanique, de l'électricité, etc...), ou ne pas en avoir, comme c'est le cas pour les modèles de comportement. Dans les deux cas, ils doivent fournir une approximation fidèle des comportements du système physique, dans la mesure où leurs paramètres sont ajustés à partir de données expérimentales. L'objectif cherché est de rendre identiques les réponses du processus et du modèle, pour des séquences d'entrée données.

Il existe plusieurs méthodes d'identification paramétrique, mais la plupart des méthodes utilisées sont basées sur le calcul du gradient pour trouver les paramètres cherchés avec une précision acceptable. Il sera raisonnable d'affirmer que les bonnes valeurs des paramètres cherchés seront celles 
qui minimisent le critère quadratique appelé souvent fonction coût. Néanmoins, il n'est pas toujours possible d'évaluer les gradients lorsque les mesures sont noyées dans le bruit. L'identification paramétrique est donc une tâche primordiale pour déterminer les valeurs numériques de paramètres d'un système pour leur utilisation dans la simulation et dans la loi de commande.

Les systèmes mécaniques sous-actionnés sont définis comme étant des systèmes dont le nombre d'actionneur est inférieur au nombre de degré de liberté [10]. On peut citer le pendule inversé, le pendulot, comme exemples des systèmes mécaniques sous-actionnés. Le manque d'actionneur complique la tâche d'identification de ce genre de système. Par conséquent, les méthodes d'identification des systèmes mécaniques complètement actionnés ne peuvent être mises en œuvre pour de tels systèmes.

Par ailleurs, le pendule inversé a fait l'objet de nombreuses études de recherche tout au long de ces dernières années. Cet intérêt est dû au fait que le pendule inversé possède plusieurs caractéristiques intéressantes entre autre la non linéarité, le couplage, l'instabilité intrinsèque [5]. Une littérature abondante traite de la commande de ce processus [3], [9], [10] et [11]. Dans [10], une commande prédictive généralisée (GPC) a été appliquée en temps-réel sur le prototype du pendule inversé stabilisé par volant d'inertie dans le but de le stabiliser autour de son point d'équilibre instable, or, l'application d'une telle commande nécessite un bon modèle du processus; ceci sous-entend donc que le processus a été identifié. Or, en ma connaissance un seul article tarite ce sujet. [5] propose une méthode d'identification neuronale du pendule inversé à l'aide d'un algorithme génétique.

Ce manuscrit présente une extension des algorithmes des moindres carrés étendus et de Steiglitz et McBride aux systèmes mécaniques sous-actionnés en utilisant les structures OE et ARMAX.

L'article est organisé comme suit: la section 2 donne un rappel sur les modèles de processus ( $\mathrm{OE}$ et ARMAX) tenant compte de la nature du bruit, et propose également une méthode de minimisation de la distance procédé-modèle pour chaque modèle. Ensuite, une modélisation en transmittance d'un modèle typique des systèmes mécanique sous-actionnés est présentée à la section 3 . Enfin, la dernière section est consacrée à la présentation des résultats d'identification et à la validation de modèles.

\section{Modèles du processus}

Par rapport aux approches non paramétriques, les méthodes paramétriques présentent un avantage considérable quant au nombre de paramètres à identifier.

L'identification des paramètres du modèle se fait sur base d'un critère d'écart entre des mesures expérimentales provenant du processus et une simulation des équations constituants le modèle.

Pour cela, il faudra choisir au préalable :

- la structure du modèle,

- le critère de performance,

- l'algorithme d'identification,

- le signal d'excitation.

Les modèles d'identification utilisent le plus souvent une représentation discrète, bien que les processus physiques réels soient généralement de nature continue. Ceci résulte du fait que, d'une part, l'ordinateur qui se charge de l'identification voit un processus discret à travers les convertisseurs $\mathrm{A} / \mathrm{N}$ et N/A et que, d'autre part, l'identification des paramètres, la synthèse du régulateur et la simulation du système sont plus simples sous forme numérique que sous forme analogique. 
Le choix de la structure du modèle dépend des hypothèses sur l'ordre du modèle du processus et la nature du bruit.

\subsection{Structures sans modèle du bruit (Ouput error)}

Cette structure est basée sur l'hypothèse selon laquelle le bruit n'est pas corrélé avec l'entrée du processus.

Supposons que la sortie mesurée du système puisse être exprimée comme suit :

$$
y(k)=G_{0}\left(q^{-1}\right) u(k)+n(k)
$$

Où $n(k)$ est un bruit stationnaire à moyenne nulle indépendant de l'entrée et $G_{0}\left(q^{-1}\right)$ est le vrai modèle du système avec une fonction de transfert d'ordre fini représentée par :

$$
G_{0}\left(q^{-1}\right)=\frac{q^{-d_{0}} B_{0}\left(q^{-1}\right)}{F_{0}\left(q^{-1}\right)}
$$

Avec

$$
\begin{aligned}
& B_{0}\left(q^{-1}\right)=b_{0}^{0}+b_{1}^{0} q^{-1}+\cdots+b_{m 0}^{0} q^{-m 0} \\
& F_{0}\left(q^{-1}\right)=1+b_{1}^{0} q^{-1}+\cdots+a_{n 0}^{0} q^{-n 0} \\
& \theta_{0}^{T}=\left[a_{1}^{0}, \cdots, a_{n 0}^{0}, b_{0}^{0}, \cdots, b_{m 0}^{0}\right]
\end{aligned}
$$

La structure du modèle présenté à l'équation (1) est connue sous le nom de la structure de l'erreur de sortie (output error, OE).

Considérons l'équation (1) en supposant être à l'instant $k-1$ et qu'on veuille prédire la sortie du système à l'instant $\mathrm{k}$ (predicteur à un pas). La sortie mesurée peut s'écrire $y(k)=y_{p}(k)+n(k)$. Il est évident que la sortie du modèle du processus $y_{p}(k)$ peut être facilement calculée avec les informations disponibles à l'instant $k-1$ à condition que les paramètres du vrai modèle $G_{0}\left(q^{-1}\right)$ soient parfaitement connus.

Donc le meilleur prédicteur pour la structure de l'erreur de sortie est :

$$
\hat{y}\left(k, \theta_{0}\right)=G\left(q^{-1}, \theta\right) u(k)
$$

Où $\hat{y}\left(k, \theta_{0}\right)$ est la sortie prédite à l'instant $\mathrm{k}$ qui dépend du vecteur de paramètres du vrai modèle $\theta_{0}$. Comme $\theta_{0}$ est en principe inconnu, on peut construire un prédicteur où le vecteur de paramètres du vrai modèle $\theta_{0}$ est remplacé par un vecteur de paramètres inconnu $\theta$ comme suit :

$$
\hat{y}(k, \theta)=G\left(q^{-1}, \theta\right) u(k)=\frac{q^{-d} B\left(q^{-1}\right) u(k)}{F\left(q^{-1}\right)} u(k)
$$

Où $G_{0}\left(q^{-1}, \theta\right)$ est l'ensemble des modèles candidats pour le vrai modèle.

La sortie prédite est en effet la sortie du modèle $y_{m}$ et l'erreur de prédiction pour cette structure est l'erreur de sortie $e_{s}(k)$.

L'erreur de prédiction s'écrit :

$$
\varepsilon(k, \theta)=\left[G_{0}\left(q^{-1}\right)-G\left(q^{-1}\right)\right] u(k)+n(k)
$$


A partir de cette équation, on observe que si $G_{0}\left(q^{-1}\right)$ appartient à l'ensemble des modèles et $\theta=\theta_{0}$, c'est-à-dire $G\left(q^{-1}\right)=G_{0}\left(q^{-1}\right)$, l'erreur de prédiction sera égale au bruit $\varepsilon(k, \theta)=n(k)$. Alors, pour $\hat{\theta}=\theta_{0}$, l'erreur de prédiction ne sera pas corrélée avec l'entrée du processus. Cette propriété intéressante peut être utilisée pour la validation du modèle identifié.

Cette structure est la meilleure structure pour identifier un modèle si le but d'identification est de trouver un modèle de simulation et que l'on n'a pas besoin d'un modèle du bruit. Cependant, les paramètres du modèle interviennent de façon non linéaire dans l'équation de l'erreur de prédiction et par conséquent cela conduit à un problème de régression non linéaire pour lequel un optimum global ne peut pas être garanti facilement.

\subsubsection{OE par la méthode de Steiglitz et Mc Bride}

Cette méthode consiste à filtrer l'entrée et la sortie par une fonction de transfert qui vaut $\frac{1}{F\left(q^{-1}\right)}$.

Pour identifier une fonction de transfert correctement, la méthode de Steiglitz et McBride minimise à chaque itération l'erreur d'équation pondérée [13].

L'OE est un système assez répandu. On définit l'erreur généralisée par :

$\varepsilon_{g}(t)=y_{f}(t)-y_{m}(t)$

${ }^{y_{f}}:$ sortie filtrée

$-u_{f}$ : entrée filtrée

On définit $y_{m}$ comme étant le modèle de sortie avec comme équation :

$y_{m}(t)=B u_{f}(t)+\left(1-F\left(q^{-1}\right)\right) y_{f}(t)=\varphi_{f}^{T}(t) \theta$

La transposé filtrée du régresser $\varphi_{f}^{T}(t)$ et le vecteur de paramètres $\theta$ sont données par :

$\varphi_{f}^{T}(t)=\left[-y_{f}(t-1) \cdots-y_{f}\left(t-n_{f}\right) u_{f}(t-1) \cdot u_{f}(t-n b)\right]$

$\theta=\left[f_{1} \cdots f_{n f} b_{1} \cdots b_{n b}\right]^{T}$.

D'ou : $\varepsilon_{g}(t)=F\left(q^{-1}\right) y_{f}(t)-B\left(q^{-1}\right) u_{f}(t)$

Si $u_{f}$ et $y_{f}$ sont respectivement l'entrée et la sortie filtrée, alors :

$u_{f}(t)=\frac{1}{F\left(q^{-1}\right)} u(t)$ et $y_{f}(t)=\frac{1}{F\left(q^{-1}\right)} y(t)$

Et donc :

$\varepsilon_{g}(t)=F\left(q^{-1}\right) y_{f}(t)-B\left(q^{-1}\right) u(t)=y(t)-\frac{B\left(q^{-1}\right)}{F\left(q^{-1}\right)} u(t)=e(t)$ 
On observe ici que l'erreur du modèle filtré est égale à l'erreur de prédiction de l'OE.

Donc en mettant à jour itérativement le regresseur linéaire on minimise l'erreur généralisée, donc l'erreur de modèle.

\subsection{Structures avec modèle du bruit (ARMAX)}

Ces structures sont basées sur l'hypothèse selon laquelle le bruit sur la sortie est un bruit blanc filtré par un filtre d'ordre fini.

L'idée est d'identifier simultanément le modèle du procédé et le modèle de la perturbation, pour pouvoir obtenir une erreur de prédiction asymptotiquement "blanche" [4].

Pour cette structure, la sortie mesurée s'écrit :

$$
y(k)=\frac{q^{-d_{0}} B_{0}\left(q^{-1}\right)}{A_{0}\left(q^{-1}\right)} u_{f}+\frac{C_{0}\left(q^{-1}\right)}{A_{0}\left(q^{-1}\right)} e(k)
$$

Cette structure, qui considère un terme de moyenne glissante (moving average) pour le bruit $C_{0}\left(q^{-1}\right) e(k)$ (quand on multiplie les deux côtés de l'équation par $A_{0}\left(q^{-1}\right)$ ) en plus du terme autorégressif, est très utilisée pour l'identification de systèmes dynamiques dans le domaine de l'automatique. Mais le prédicteur basé sur cette structure est non linéaire par rapport au vecteur des paramètres à identifier [15].

Comme les deux fonctions de transfert possèdent le même dénominateur, ce modèle est utile lorsque le bruit agit en amont du processus.

L'équation de prédiction de la sortie pour le modèle ARMAX est :

$$
\hat{y}(k)=\frac{q^{-d} B\left(q^{-1}\right)}{A\left(q^{-1}\right)} u(k)+\left[\frac{C\left(q^{-1}\right)}{A\left(q^{-1}\right)}-1\right] \varepsilon(k)
$$

L'erreur de prédiction pour le modèle ARMAX s'écrit donc :

$$
\varepsilon(k)=\frac{A\left(q^{-1}\right)}{C\left(q^{-1}\right)}\left[y(k)-\frac{q^{-d} B\left(q^{-1}\right)}{A\left(q^{-1}\right)}\right] u(k)
$$

L'erreur de prédiction est non linéaire par rapport au paramètres du modèle du processus et du bruit. L'erreur de prédiction sera blanche si la structure du vrai modèle est ARMAX et appartient à l'ensemble des modèles candidats.

\subsubsection{ARMAX par moindres carrés étendus}

Rappelons d'abord que la structure ARMAX rajoute un jeu de coefficients, provenant de l'ajout d'un numérateur au filtre du bruit. Donc ce type d'identification est plus compliqué, moins stable numériquement par ce que plus on a des coefficients à identifiés plus on a besoin de mesures pour arriver à capturer les dynamiques du système, et à ceci rajouter le fait que la régression n'est pas linéaire.

Quelle que soit la procédure d'identification, la fonction erreur ne sera en général pas nulle à cause des erreurs de caractérisation et des perturbations de mesure. La validation d'un modèle passe par la minimisation de la distance Objet-Modèle prédéfinie [14] et [15]. L'article [4], parle de la méthode de minimisation de l'erreur quadratique par moindre carré étendu (MCE), dont nous rappelons les équations permettant sa mise en œuvre : 


$$
\begin{aligned}
& \varepsilon(t)=y(t)-\hat{\theta}^{T}(t-1) \varphi(t) \\
& \hat{\theta}(t)=\hat{\theta}(t-1)+p(t) \varphi(t) \varepsilon(t) \\
& p(t)=p(t-1)-p(t-1) \frac{\varphi(t) \varphi^{T}(t) p(t-1)}{1+\varphi^{T}(t) p(t-1) \varphi(t)}
\end{aligned}
$$

Où :

- $\theta^{T}$ : vecteur des paramètres à identifier

- $\varphi^{T}(t)$ : vecteur des données.

$-\quad y(t):$ sortie du système

$-\varepsilon(t):$ erreur de prédiction

- $p(t)$ : matrice (posé pour éviter de calculer une inverse de matrice à chaque pas)

La méthode MCE reste la même que la méthode des moindres carrés récursif, sauf que la matrice $\Phi_{N}$ et le vecteur $\theta$ sont modifiés. On estime récursivement les erreurs de prédiction grâce à un algorithme des moindres carrés récursif.

\section{Modélisation du pendule inverse}

Une littérature abondante traite de la modélisation de type représentation d'état du pendule inversé. Dans notre cas nous nous intéresserons à trouver la fonction de transfert de ce système en partant des équations différentielles qui le régissent.

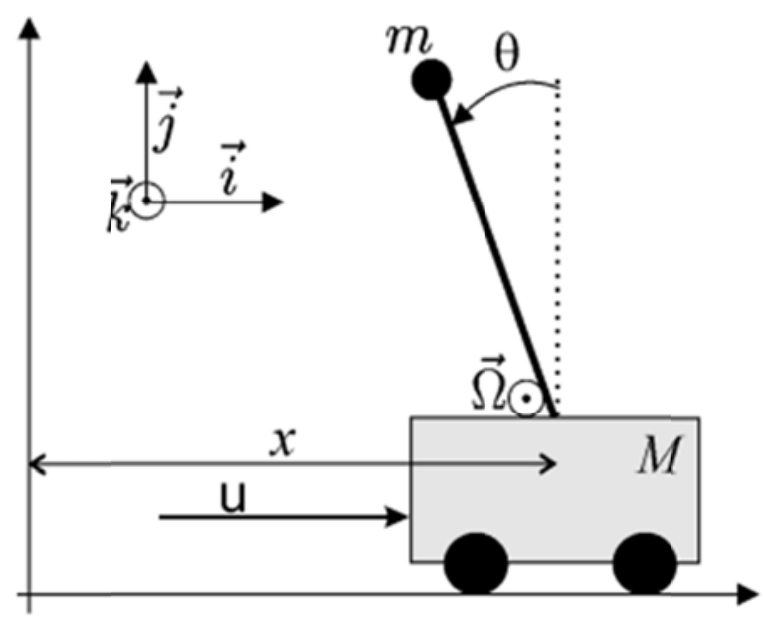

Figure 1. Pendule inversé

Considérons le pendule inversé formé d'un pendule de masse $\mathrm{m}$ posé en équilibre instable sur un chariot roulant comme représenté sur la figure 1. La quantité $u$ est la force exercée sur le chariot de masse $M, x$ indique la position du chariot, $\theta$ est l'angle entre le pendule et la verticale [12].

Dans [3], on établit les équations du mouvement en utilisation les équations d'Euler-Lagrange, dont nous reprenons ici les résultats.

Les équations du mouvement du pendule sont données par : 
$\left\{\begin{array}{l}(M+m) \frac{d^{2} x}{d t^{2}}+m l \frac{d^{2} \theta}{d t^{2}} \cos \theta-m l \frac{d \theta}{d t} \sin \theta=u \\ \left(m l \frac{d^{2} x}{d t^{2}} \cos \theta+m l^{2} \frac{d^{2} \theta}{d t^{2}}-m g l \sin \theta=0\right.\end{array}\right.$

Ces équations sont non linéaires. Le système linéarisé au point d'équilibre est donné par :

$\left\{\begin{array}{l}(M+m) \frac{d^{2} x}{d t^{2}}+m l \frac{d^{2} \theta}{d t^{2}}=u \\ \left(\frac{d^{2} x}{d t^{2}}+l \frac{d^{2} \theta}{d t^{2}}-g \theta=0\right.\end{array}\right.$

En choisissant $\theta$ l'angle entre le pendule et la verticale comme sortie du système sachant que $u$ est l'entrée, et en appliquant la transformé de Laplace, on exprime la fonction de transfert du système par :

$G(p)=\frac{-1}{M l p^{2}+(M+m) g}$

\section{Simulation et validation}

Pour valider notre approche d'identification on partira d'un modèle connu, ensuite on généra des données à partir de ce système connu. On utilise enfin les outils d'identification pour reconstruire le modèle de départ.

Pour illustrer la méthode, on considère la fonction de transfert du pendule avec comme valeurs de coefficients :

$$
\begin{aligned}
& -\quad m=1 \mathrm{~kg} \\
& -\quad M=5 \mathrm{~kg} \\
& -\quad g=10 \mathrm{~m} / \mathrm{s}^{2} \\
& -\quad l=1 \mathrm{~m}
\end{aligned}
$$

Les essais sont réalisés avec un bruit de mesure blanc puis coloré. L'acquisition des données entréesortie se fait sur 10000 échantillons avec une période d'acquisition de $100 \mathrm{~ms}$.

Le signal d'entée choisi est une séquence binaire pseudo-aléatoire (SBPA). En effet, une démarche classique en identification stipule que l'obtention d'un modèle identifié reflétant le plus fidèlement possible le comportement du système nécessite que ce système soit sollicité par une entrée relativement riche, de façon à exciter le plus grand nombre de modes propres. Le meilleur signal candidat est alors le bruit blanc, malheureusement non déterministe. Une séquence binaire pseudo-aléatoire est un signal déterministe à approcher d'une réalisation d'un bruit blanc [1].

\subsection{Identification en présence du bruit blanc}

On considère ici le cas où le bruit est blanc. Dans un tel contexte le modèle approprié pour l'identification est donc un modèle à erreur de sortie. Pour ne pas surdimensionné la structure, le principe de parcimonie veut qu'on commence par la structure du modèle la plus simple.

En simulant le système avec un bruit sur la mesure de $10 \%$ de variance et en utilisant comme modèle du processus un ARX avec comme critère d'optimisation la méthode des moindres carrés on récursif, on obtient comme fonction de transfert du système : 
La figure 2 compare la réponse indicielle du modèle et celle du processus. On observe un biais dans cette estimation, évidemment par ce que l'ARX n'est pas la bonne structure du modèle pour ce type de bruit.

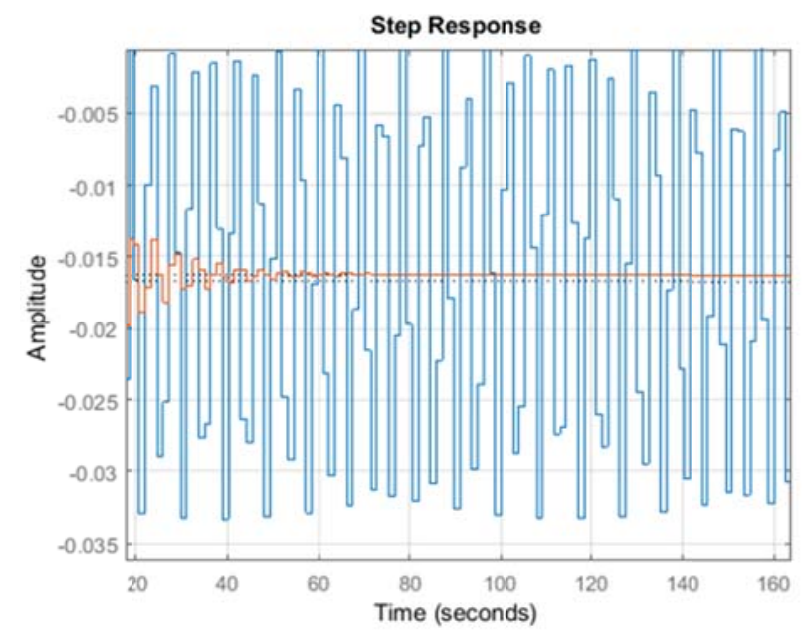

Figure 2. Réponse indicielle du modèle et du processus

La meilleure structure pour ce type de bruit est l'OE. Signalons également que l'ARMAX peut filtrer ce type de bruit; en effet un OE est un ARMAX où $C(q)=A(q)$. Mais utiliser un ARMAX serait surdimensionné la structure du modèle en ajoutant des pôles et des zéros dans la fonction de transfert.

En vue de savoir si l'algorithme des moindres carrés appliquée au modèle OE permet de capturé les dynamiques du système d'une part et de savoir si les résidus sont blancs et non corrélés avec le signal d'entrée d'autre part, les figures 3 et 4 comparent respectivement les sorties du modèle et du processus et examine les résidus du modèle identifié. On observe un net biais dans cette estimation dû au fait que le bruit n'est pas blanc. Ceci montre également les limites de cet algorithme des moindres carrés non récursif dans l'impossibilité de capturer les dynamiques du système.

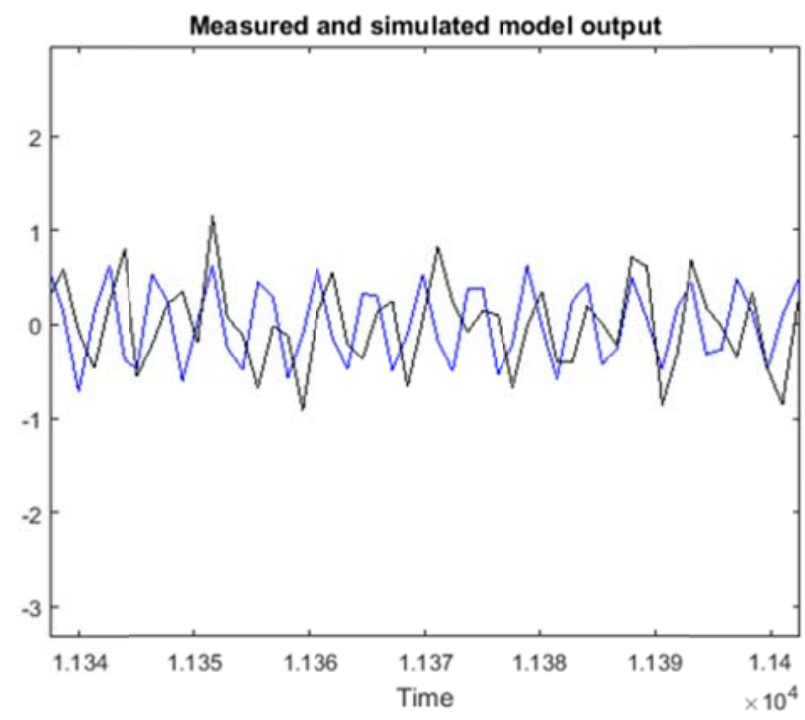

Figure 3. Sortie du modèle et du processus 

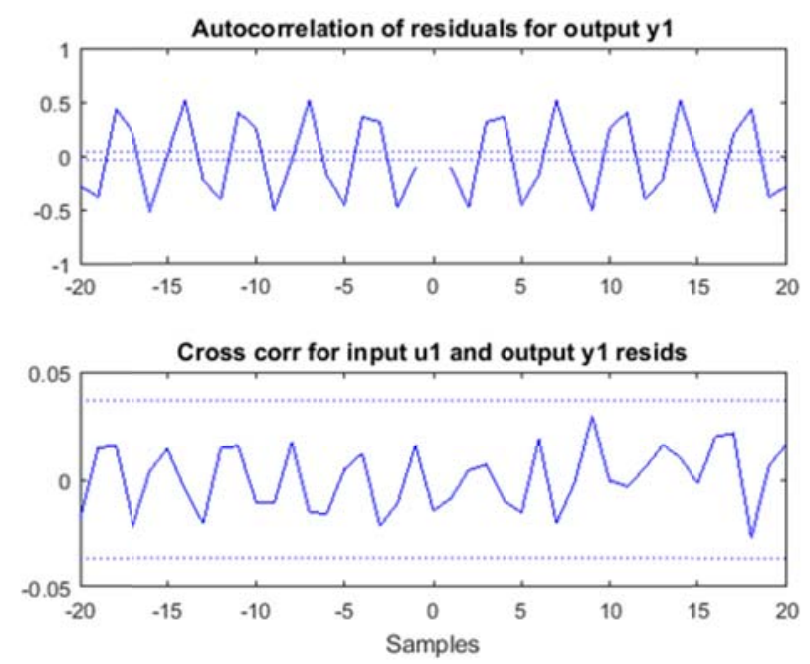

Figure 4. Test de corrélation pour le modèle identifié

En appliquant l'algorithme de Steiglitz et Mc Bride, on obtient dès la première itération une nette amélioration pour le suivi de la dynamique du système comme l'illustre la figure 5. Cette amélioration est dû essentiellement au fait que cet algorithme permet de filtrer en même temps l'entrée et la sortie du système.

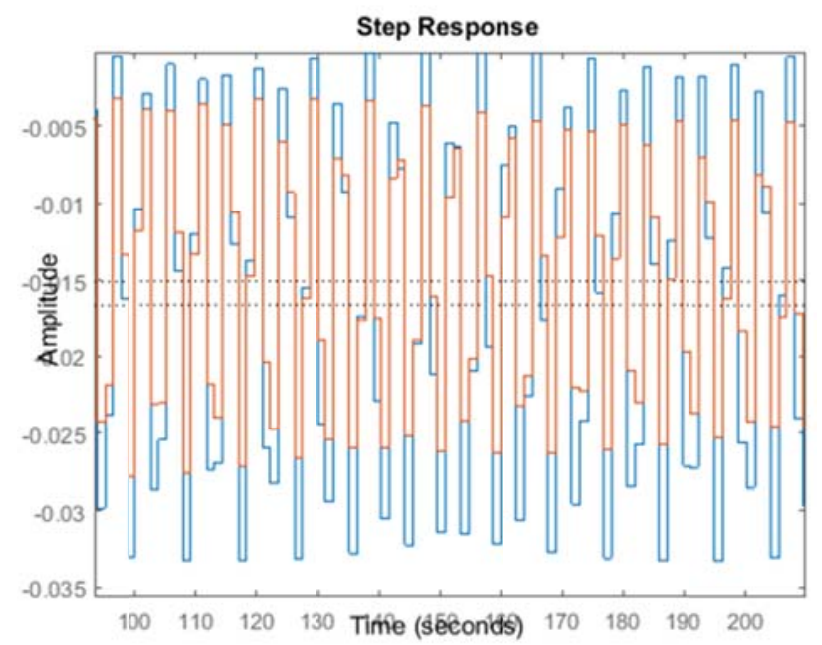

Figure 5. Réponses indicielles de la première itération

On observe une convergence des paramètres à partir de la seconde itération; la fonction de transfert est :

$\frac{-0.01997 z^{-1}-0.01782 z^{-2}}{1+0.4132 z^{-1}+0 . .9999 z^{-2}}$

Cet algorithme permet d'obtenir une convergence rapide des paramètres du système.

Pour également valider cette approche nous comparons les diagrammes de Bode du processus et du modèle. On remarque sur la figure 6 que les deux diagrammes coïncident assez parfaitement et que les régions de confiance sont minimisées. 


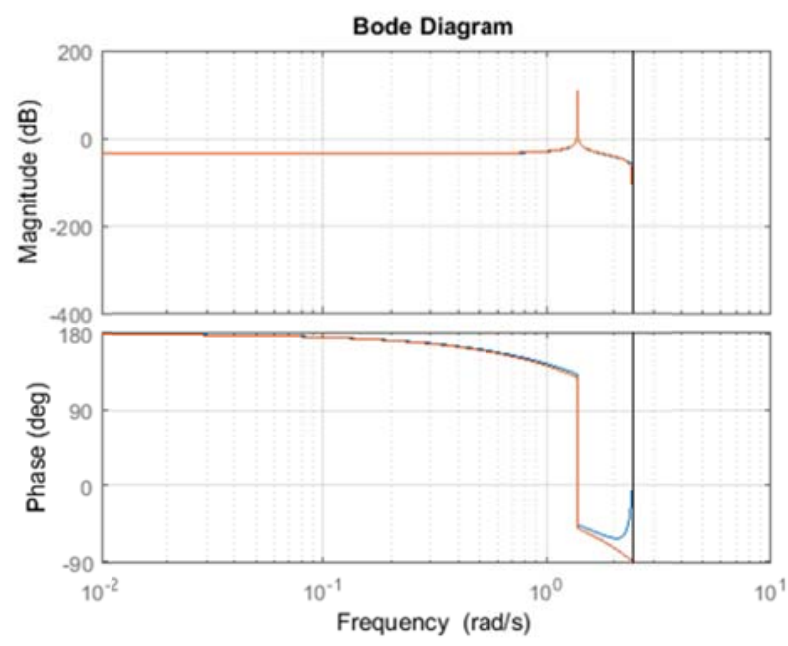

Figure 6. Diagrammes de Bode du modèle et du processus

\subsection{Identification en présence d'un bruit coloré}

On considère dans cette section le cas où le bruit est coloré.

Le bruit étant indépendant de l'entrée, l'application d'une méthode à erreur de sortie, comme dans la section précédente, continue de fournir une estimation non biaisée des paramètres de $G(p)$ dès lors que la structure de cette fonction de transfert est respectée dans le modèle. En revanche, le bruit étant coloré, on observe que l'ellipsoïde de confiance est plus grande avec une telle méthode qu'avec une méthode respectant la nature du bruit, c'est à dire ARMAX [7].

Sur la figure 7 on remarque qu'en testant la qualité du prédicteur à un pas à partir de l'itération 100 , l'erreur tend asymptotiquement vers un bruit blanc, ce qui garantit une estimation non biaisée des paramètres.

Les différents tests de validation montrent l'aptitude du modèle flou globale obtenu à reproduire les dynamiques des sorties de l'échangeur de chaleur, et ce malgré les bruits de mesures.

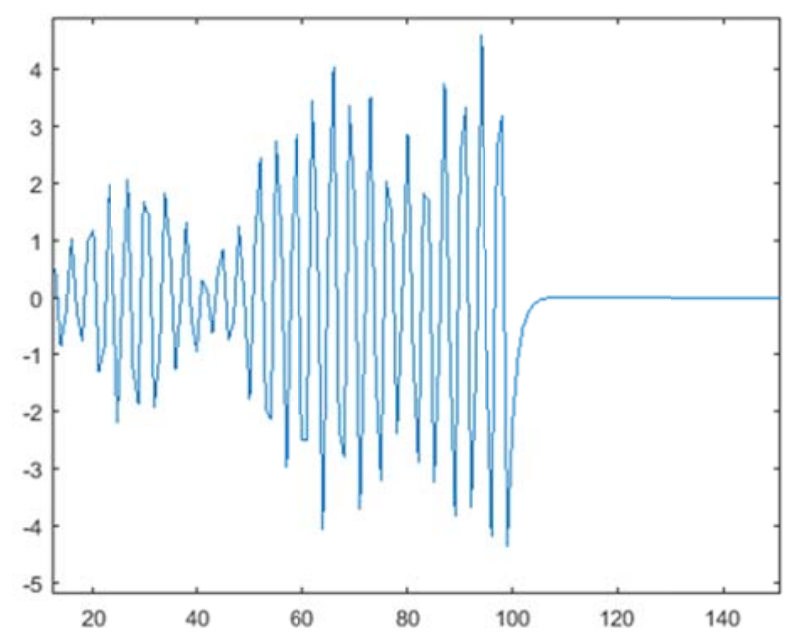

Figure 7. Erreur de prédiction testé à l'itération 100

La figure 8 , représente et compare la sortie du modèle simulé avec la sortie mesurée en vue de savoir si les dynamiques du système ont bien été estimées.

On remarque malgré la présence d'un bruit de $10 \%$ de variance sur la mesure, la sortie du modèle capture les dynamiques du système. 


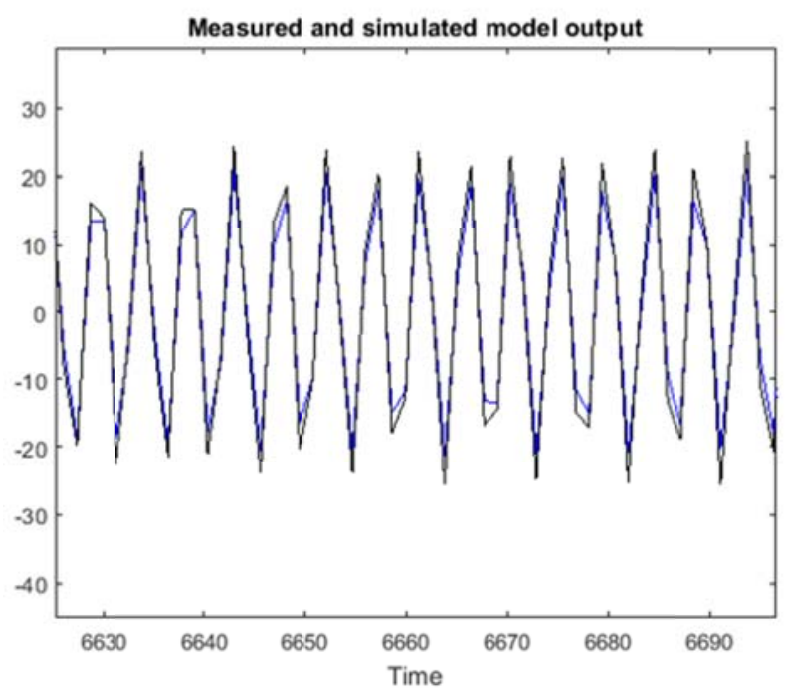

Figure 8. Sorties du modèle et du processus

Il est également possible d'examiner les résidus (erreur de simulation) de ce modèle, puis de représenter l'autocorrélation des résidus et l'inter corrélation entre l'entrée et les résidus.

Disons que l'analyse des résidus est essentielle pour la validation des paramètres estimés. L'approche classique repose sur les fonctions normalisées d'inter corrélation et d'autocorrélation qui permettent d'établir successivement l'hypothèse de blanchissement de l'erreur de prédiction résiduelle et l'hypothèse de son indépendance vis-à-vis de l'entrée, afin de détecter d'éventuelles corrélations qui invalideraient le modèle [7].

D'après la figure 9 , on peut remarquer que les résidus sont blancs et totalement non corrélés avec le signal d'entrée. Le modèle estimé est donc satisfaisant.
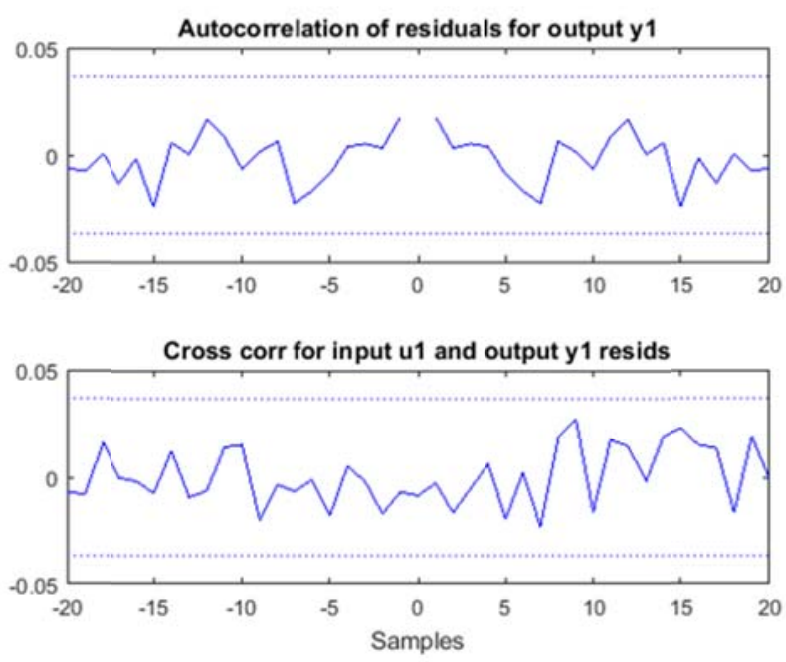

Figure 9. Test de corrélation pour le modèle identifié

Pour terminer cette étape de validation comparons les diagrammes de Bode du modèle estimé et du processus. A la figure 10, on peut observer que les diagrammes de Bode du modèle coïncident très bien avec celui du processus et que les régions de confiance sont très petites. 


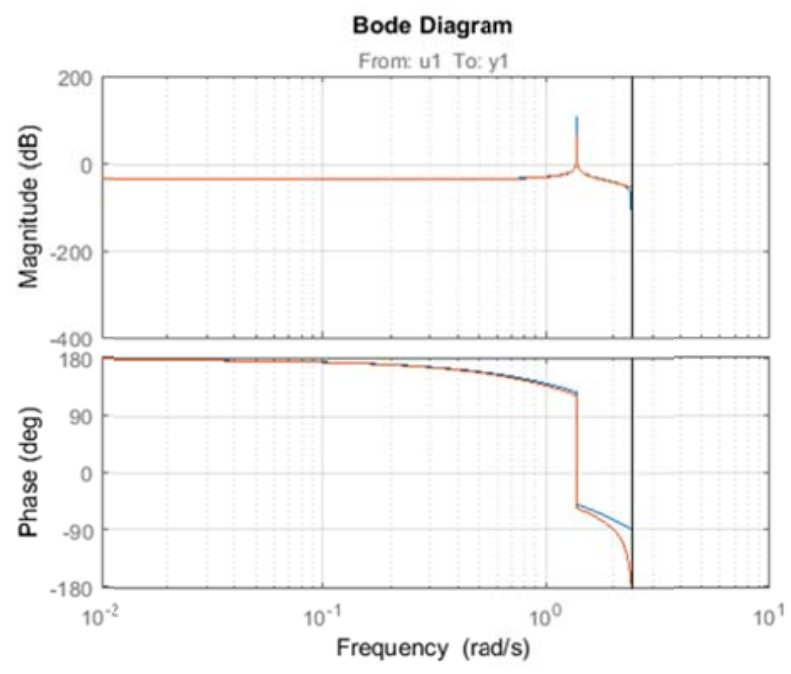

Figure 10. Diagrammes de Bode du modèle et du processus

Le modèle ARMAX du processus identifié et validé est :

$\left\{\begin{array}{l}A(z)=1+0.415 z^{-1}+z^{-2} \\ B(z)=-0.01708 z^{-1}-0.01994 z^{-2} \\ C(z)=1-0.5179 z^{-1}+0.00324 z^{-2}\end{array}\right.$

\section{Conclusion}

Deux méthodes d'identification récursive des systèmes mécaniques sous-actionnés sont présentées. Il s'agit d'une extension des algorithmes des moindres carrés étendus et de Steiglitz et McBride au cas des systèmes sous-actionnés. Les modèles utilisés sont de type OE et ARMAX. Bien que ces méthodes présentent l'avantage de suivre l'évolution des paramètres dans le cas des systèmes variants dans le temps, l'analyse par simulation numérique d'un modèle typique nous a montré les performances caractéristiques de ces méthodes par rapport à celles obtenues en utilisant la méthode des moindres carrés linéaires non récursifs. Ces performances sont considérablement améliorées en utilisant des modèles OE et ARMAX respectivement en présence d'un bruit blanc et coloré.

\section{Références}

[1] F.Lamnabhi-Lagarrigue et P.Rouchon, systèmes non linéaires, Paris, Hermès Science Publications, 2002

[2] B.Salemi, B.Remy, A.Degiovanni, F.Uriz et O.Verseux. Mise en place de modèles paramétriques autorégréssif de type ARX pour l'extrapolation de températures d'équipements dans la nacelle d'un système propulsif, Congrés Français de Thermique, SFT 2011

[3] E.Trélat, Contrôle optimale theorie et applicatons, Vuibert-Avril 2005

[4] A.Amaddi, A.Guessoum, D.Berkani, O.Belkina, Etude de la méthode des moindres carrés étendus et application au signale de parole, Conférences:Science of electronic, Tunis 2005

[5] S.Dallal, Identification paramétrique d'un pendule inversé simple par un algorithme génétique, Université Mohamed Khider Biskra, mémoire de Master, Algér 2014.

[6] E.Pillet, S.Cogan et N.Bouhaddi. Identification des paramètres de conception à partir de mesures incertaines, Colloque National en calcul des structures, France, Giens, 2005

[7] H.Bourlès et P.Gabot. Identification des systèmes linéaires dans une formulation mixte continue/discrète,APII-JESA, 2001

[8] S.Avril et F.Pierron. Identification à partir de mesures de champs,Colloque national en calcul des structures, France, Giens 2007 
[9] S.Riachy, T.Floquet et JP.Richard. Stabilisation de systèmes mécaniques sous-actionnés par commande quasihomogène, e-STA, Revue électronique Science et Technologies de l'Automatique, http://www.see.asso.fr/esta, Vol. 3, No. 3, 2007.

[10] A.Chemori, S.Krut, N.Touati. Le pendule inversé stabilisé par volant d'inertie, 3èmes Journées Démonstrateurs, Angers, France, 2010

[11] H.Wang, C.Vasseur, V.Koncar, A.Chamroo. Modélisation et commande d'un pendule inversé sur un robot X-Y par asservissement visuel. Université de poitiers, France, 2009

[12] L.Jaulin, Mobile robotics, ISTE Press - Elsevier, Octobre 2015

[13] M.Ashari, M.Mboup, P.A.Regalia. Sur les points stationnaires de la méthode de Steiglitz et McBride dans le cas sous modélisé. Quinzième colloque GRETSI, Juan-Les-Pins, 1995.

[14] M.Zegrari, A.Badri et B.Oukarfi. Identification par la méthode du modèle des paramètres d'une machine à courant continu. $3^{\text {nd }}$ International Conference of Electronic,Technologies of Information and Telecommunications, SETIT, Tunis, March 2005

[15] P.Larminat. Analysis and control of linear systems. ISTE Press - Elsevier, January 2007. 